\title{
Magneto-optic and transverse-transport properties of noncollinear antiferromagnets
}

\author{
Sebastian Wimmer $\odot,{ }^{1, *}$ Sergiy Mankovsky, ${ }^{1}$ Ján Minár, ${ }^{2}$ Alexander N. Yaresko, ${ }^{3}$ and Hubert Ebert ${ }^{1}$ \\ ${ }^{1}$ Department Chemie, Physikalische Chemie, Universität München, Butenandtstr. 5-13, 81377 München, Germany \\ ${ }^{2}$ New Technologies Research Centre, University of West Bohemia, Univerzitni 8, 30614 Pilsen, Czech Republic \\ ${ }^{3}$ Max-Planck-Institute for Solid State Research, Heisenbergstrasse 1, 70569 Stuttgart, Germany
}

(Received 20 September 2019; revised manuscript received 26 November 2019; published 23 December 2019)

\begin{abstract}
Previous studies on the anomalous Hall effect in coplanar noncollinear antiferromagnets are revisited and extended to magneto-optic properties, namely magneto-optic Kerr effect (MOKE) and x-ray magnetic dichroism (XMCD). Starting from group-theoretical considerations the shape of the frequency-dependent conductivity tensor for various actual and hypothetical spin configurations in cubic and hexagonal $\mathrm{Mn}_{3} X$ compounds is determined. Calculated MOKE and x-ray dichroism spectra are used to confirm these findings and to give estimates of the size of the effects. For $\mathrm{Mn}_{3} \mathrm{IrPt}$ and $\mathrm{Mn}_{3} \mathrm{PtRh}$ alloys the concentration dependence of the anomalous and spin Hall conductivity is studied in addition.
\end{abstract}

DOI: 10.1103/PhysRevB.100.214429

\section{INTRODUCTION}

The anomalous Hall effect (AHE) in magnetically ordered materials is usually considered to scale with the corresponding magnetization [1-4]. The same applies to the magneto-optical Kerr effect [5,6] (MOKE) that is used in particular to monitor the magnetization dynamics [7,8]. While the linear relationship of the AHE and the MOKE with the magnetization appears plausible, there seems to be no strict formal justification given for this in the literature apart from numerical studies based on ab initio calculations [9]. Nevertheless, a sum rule has been derived that relates the integrated off-diagonal optical conductivity to the orbital magnetic moment of the material $[10,11]$. The magnetic circular dichroism in $\mathrm{x}$-ray absorption (XMCD) is very closely related to the MOKE [12]. To make full use of this local magnetic probe several authors have derived the so-called XMCD sum rules [13-15] that allow for an example to deduce from the integrated $\mathrm{L}_{2,3^{-}}$ spectra of 3d-transition metals their spin and orbital magnetic moments. In line with the sum rules an angular dependency according to $\cos (\hat{m} \cdot \hat{q})$ is assumed, where $\hat{m}$ and $\hat{q}$ are the orientation of the local moment probed by XMCD and that of the incident $\mathrm{x}$-ray beam. This simple relation implies in particular that in spin-compensated antiferromagnetic systems the XMCD should vanish. For such systems information on the local magnetic moment can nevertheless be obtained by exploiting the linear magnetic x-ray dichroism (XMLD) for which two spectra with linear polarization parallel and perpendicular to the local magnetization are recorded [16].

In contrast to this situation, Chen et al. [17] pointed out that perfect spin compensation in an antiferromagnet is not a sufficient criterion for the AHE to be absent. In fact these authors give symmetry arguments that the AHE can indeed occur for example in spin-compensated noncollinear antiferromagnets as $\mathrm{Mn}_{3} \mathrm{Ir}$ that is commonly employed in spin-valve devices. Numerical work by Chen et al. for $\mathrm{Mn}_{3} \mathrm{Ir}$ gives in fact a rather

\footnotetext{
*sebastian.wimmer@cup.uni-muenchen.de
}

large anomalous Hall conductivity, comparable in size to the values for Fe, Co, and Ni. Kübler and Felser [18] numerically confirmed the results of Chen et al. and investigated in addition the noncollinear antiferromagnetic hexagonal compounds $\mathrm{Mn}_{3} \mathrm{Ge}$ and $\mathrm{Mn}_{3} \mathrm{Sn}$ considering various coplanar and noncoplanar triangular magnetic configurations. Also in this case several spin-compensated configurations were identified that were predicted to exhibit an AHE. Recently, a large AHE and MOKE have been predicted also for the noncollinear AFMs $\mathrm{Mn}_{3} X \mathrm{~N}(X=\mathrm{Ga}, \mathrm{Zn}, \mathrm{Ag}$, and $\mathrm{Ni})$ via first-principles density functional calculations together with group-theory analysis and tight-binding modeling [19]. These predictions recently could indeed be experimentally verified in $\mathrm{Mn}_{3} \mathrm{Sn}$ [20] and $\mathrm{Mn}_{3} \mathrm{Ge}[21,22]$.

Here one should stress that the occurrence of off-diagonal antisymmetric conductivity tensor elements has been unambiguously determined much earlier. In particular Kleiner [23] gave the space-time symmetry-restricted tensor forms for the electrical conductivity in all magnetic solids based on the transformation properties of the corresponding Kubo formula under the symmetry operations of the relevant magnetic group. These results in particular do not rest on the assumption of collinear magnetic order or a finite magnetization. Moreover, they apply to the frequency-dependent or optical conductivity just as well and therefore imply the presence of MOKE as well as XMCD signals concomitant with the AHC. The former has been studied in chiral cuprates based on tight-binding model calculations of the Berry curvature [24] and, using first-principles methods, in cubic $\mathrm{Mn}_{3}$ Ir-type compounds [25]. The symmetry criteria for the presence of the spin Hall effect (SHE) have recently been given by the present authors [26], extending the approach by Kleiner [23] to more complex situations. The SHE in noncollinear antiferromagnets is currently the subject of intensive theoretical efforts [27-30] and has been observed in experiments on $\mathrm{Mn}_{3} \operatorname{Ir}$ [31] via its contribution to the so-called spinorbit torque. Due to the identical transformation properties of electronic charge and heat current operators $[23,26]$ the 
occurrence of AHE and SHE imply the existence of corresponding thermally-induced effects, the anomalous and spin Nernst effects. These have been studied in hexagonal $\mathrm{Mn}_{3} X$ $(X=\mathrm{Sn}, \mathrm{Ge}, \mathrm{Ga})$ using the Berry curvature approach and a Mott-like formula [32]. The anomalous Nernst effect has recently been observed experimentally in $\mathrm{Mn}_{3} \mathrm{Sn}$ [33]. Of particular current interest in the thriving field of noncollinear magnetism is the occurrence of chirality-induced or so-called topological effects arising from the emergent electromagnetic field created by a nontrivial real- or reciprocal-space texture [34]. This will be the subject of another publication [35].

In the following we present a theoretical study on the transverse-transport and optical properties of noncollinear coplanar antiferromagnets. The next section (II) gives a brief overview on the theoretical framework and methods applied. The major part of this contribution is devoted to results for the cubic prototype system $\mathrm{Mn}_{3} \mathrm{Ir}$ in Sec. III and a number of possible magnetic configurations of hexagonal $\mathrm{Mn}_{3} \mathrm{Ge}$ in Sec. IV.

\section{THEORETICAL FRAMEWORK}

\section{A. Magnetic symmetry and shape of the conductivity tensor}

For the specific case of $\mathrm{Mn}_{3} \mathrm{Ir}$ the occurrence of a nonvanishing AHE was predicted by Chen et al. [17] by explicitly considering kagome-type sublattices occupied by Mn atoms with triangular antiferromagnetic order (see below) in combination with a suitable model Hamiltonian. A more general scheme to search for a finite AHE in spin-compensated systems is to use Kleiner's tables that give among others the shape of the frequency dependent conductivity tensor $\sigma(\omega)$ for any material [23]. These tables were constructed by starting from Kubo's linear response formalism and making use of the behavior of the current density operator under all symmetry operations of the relevant magnetic space group. It turns out that only the magnetic Laue group of a material has to be known to fix unambiguously the shape of $\sigma(\omega)$. Unfortunately, Kleiner [23] used an old definition for the Laue group that is obtained by removing the inversion from all improper symmetry operations of the magnetic point group leading to the magnetic Laue Group 32' in the case of $\mathrm{Mn}_{3} \operatorname{Ir}$ [23]. With the (magnetic) Laue group defined [36] to be the (magnetic) point group artificially extended by the inversion one is led to the magnetic Laue group $\overline{3} m^{\prime}$ instead. Kleiner's tables have been updated recently by Seemann et al. [26] to account in particular for the revised definition of the magnetic Laue group.

Having determined the magnetic Laue group of a solidfor this purpose the program FINDSYM [37,38] was used here-the specific shape of its conductivity tensor $\sigma(\omega)$ can be read from these tables. The presence of an antisymmetric part for the off-diagonal tensor elements in particular indicates the simultaneous occurrence of the AHE, the MOKE, as well as the XMCD (see below).

\section{B. First-principles calculations of the anomalous Hall effect, the magneto-optical Kerr effect, and the x-ray magnetic dichroism}

The qualitative investigations on the occurrence of the AHE, the MOKE, and XMCD of various materials presented below had been complemented by corresponding numerical work. The underlying electronic structure calculations have been done within the framework of relativistic spin density functional theory (SDFT) with the corresponding Dirac Hamiltonian given by [39]:

$$
\mathcal{H}_{\mathrm{D}}=c \boldsymbol{\alpha} \cdot \vec{p}+\beta m c^{2}+V(\vec{r})+\beta \vec{\Sigma} \cdot \vec{B}_{\mathrm{xc}}(\vec{r}) .
$$

Here all quantities have their usual meaning as used in the four-component Dirac formalism [40]. Within SDFT $\vec{B}_{\mathrm{xc}}(\vec{r})$ stands for the spin-dependent part of the exchange correlation potential and of course reflects the noncollinear spin configuration of the system. In practice, a unique orientation of the magnetization along $\hat{e}_{z^{\prime}}$ is assumed in general within an atomic cell, leading to the expression $\vec{B}_{\mathrm{xc}}(\vec{r})=B_{\mathrm{xc}}(\vec{r}) \hat{e}_{z^{\prime}}$. This allows us to solve the Dirac equation very efficiently for a local frame of reference with the coupling term simplified to $\beta \Sigma_{z^{\prime}} B_{\mathrm{xc}}(\vec{r})$ [41]. For the calculations presented here the spin-averaged and spin-dependent exchange correlation contributions $\bar{V}_{\mathrm{xc}}(\vec{r})$ and $B_{\mathrm{eff}}(\vec{r})$, respectively, to the effective potential $V(\vec{r})$ were set up using the parametrization of Vosko et al. [42]. To deal with the resulting four-component Dirac equation the spin-polarized relativistic (SPR) formulations of the Korringa-Kohn-Rostoker (KKR) $[43,44]$ and linearmuffin-tin-orbital (LMTO) [45-47] methods have been used. With the electronic Green function $G^{+}\left(\vec{r}, \vec{r}^{\prime}, E\right)$ supplied by the SPR-KKR method the dc-conductivity tensor $\sigma$ has been evaluated on the basis of the Kubo-Středa equation $[48,49]$ :

$$
\begin{aligned}
\sigma_{\mu \nu}= & \frac{\hbar}{4 \pi N \Omega} \operatorname{Trace}\left\langle\hat{j}_{\mu}\left(G^{+}-G^{-}\right) \hat{j}_{\nu} G^{-}-\hat{j}_{\mu} G^{+} \hat{j}_{\nu}\left(G^{+}-G^{-}\right)\right\rangle_{\mathrm{c}} \\
& +\frac{e}{4 \pi i N \Omega} \operatorname{Trace}\left\langle\left(G^{+}-G^{-}\right)\left(\hat{r}_{\mu} \hat{j}_{\nu}-\hat{r}_{\nu} \hat{j}_{\mu}\right)\right\rangle_{\mathrm{c}},
\end{aligned}
$$

where the indices $\mu$ and $v$ stand for the Cartesian coordinates $x, y$, or $z$, while $G^{+}$and $G^{-}$represent the retarded and advanced, respectively, Green function to be evaluated at the Fermi level $E_{\mathrm{F}}$.

Application of Eq. (2) for disordered systems, as implied here by the substitutional disorder on the $5 \mathrm{~d}$ sublattice, requires an appropriate averaging over all configurations as indicated by the notation $\langle\ldots\rangle_{\mathrm{c}}$. Within the present work the CPA (Coherent Potential Approximation) alloy theory has been used for this purpose that allows in particular a proper treatment of the resulting vertex corrections needed to handle terms like $\left\langle\ldots G^{+} \ldots G^{-}\right\rangle_{\mathrm{c}}$ [50]. Concerning further technical details of the implementation of Eq. (2), see for example Ref. [51].

The optical conductivity tensor $\sigma(\omega)$ for finite frequencies, on the other hand, has been determined using the SPR-LMTO method and the standard expression for the absorptive part of the diagonal and off-diagonal tensor elements, $\sigma_{\mu \mu}^{1}(\omega)$ and $\sigma_{\mu \nu}^{2}(\omega)$, respectively [12,52,53]:

$$
\begin{gathered}
\sigma_{\mu \mu}^{1}(\omega)=\frac{\pi e^{2}}{\hbar \omega m^{2} V} \sum_{\substack{j^{\prime} \cdot \mathrm{kcc} . \\
j \vec{k} \text { unocc. }}}\left|\Pi_{j j^{\prime}}^{\mu}\right|^{2} \delta\left(\omega-\omega_{j j^{\prime}}\right) \\
\sigma_{\mu \nu}^{2}(\omega)=\frac{\pi e^{2}}{\hbar \omega m^{2} V} \sum_{\substack{j^{\prime} \vec{k} \text { occ. } \\
j \vec{k} \text { unocc. }}} \Im\left(\Pi_{j^{\prime} j}^{\mu} \Pi_{j j^{\prime}}^{\nu}\right) \delta\left(\omega-\omega_{j j^{\prime}}\right)
\end{gathered}
$$




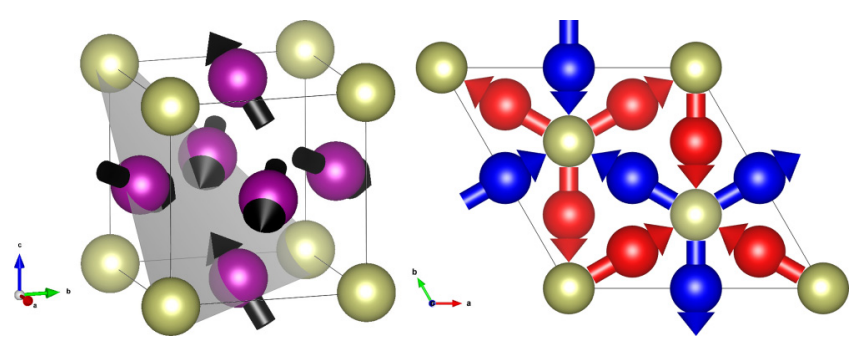

FIG. 1. Left: Cubic unit cell of $\mathrm{Mn}_{3} \mathrm{Ir}$ in the triangular antiferromagnetic structure. Ir atoms are colored in gold (light gray), Mn atoms in purple (dark gray). Black arrows indicate the direction of the magnetic moments on the Mn sites, the gray-shaded surface marks the (111) plane. Right: View along [111] on the kagome planes; $\mathrm{Mn}$ atoms and moments in alternating layers are colored in red and blue [57].

with the matrix elements $\Pi_{j^{\prime} j}^{\mu}=\left\langle\phi_{j^{\prime} \vec{k}}\left|\mathcal{H}_{\mu}\right| \phi_{j \vec{k}}\right\rangle$ of the Bloch states $\left|\phi_{j \vec{k}}\right\rangle$ and their energy difference $\omega_{j j^{\prime}}=E_{j \vec{k}}-E_{j^{\prime} \vec{k}}$. The indices $\mu$ and $v$ again represent the polarization using the Cartesian coordinates $x, y$, and $z$. The dispersive part was determined in a second step by means of a KramersKronig transformation. With the full tensor $\sigma(\omega)$ available, the Kerr rotation angle $\theta_{\mathrm{K}}$ was obtained from the standard expression [12,53]:

$$
\theta_{\mathrm{K}} \simeq \Re\left(\frac{\sigma_{\mathrm{xy}}(\omega)}{\sigma_{\mathrm{xx}}(\omega) \sqrt{1-\frac{4 \pi i}{\omega} \sigma_{\mathrm{xx}}(\omega)}}\right),
$$

that clearly shows that the Kerr rotation-as the Kerr ellipticity-is directly connected with the off-diagonal element $\sigma_{\mathrm{xy}}(\omega)$ of the conductivity tensor.

Finally, the x-ray absorption coefficients $\mu_{\lambda}(\omega) f$ for circularly polarization $\lambda$ have been calculated using the SPR-KKR Green function method on the basis of the expression [12,54]:

$$
\mu_{\lambda}(\omega) \propto \sum_{i \text { occ }}\left\langle\Phi_{i}\left|\mathcal{H}_{\lambda}^{\dagger} \Im G^{+}\left(E_{i}+\hbar \omega\right) \mathcal{H}_{\lambda}\right| \Phi_{i}\right\rangle \theta\left(E_{i}+\hbar \omega-E_{\mathrm{F}}\right),
$$

where the functions $\Phi_{i}$ represent the probed core states $i$ at energy $E_{i}$ and $E_{\mathrm{F}}$ is the Fermi energy. It should be noted that this expression is in line with Eqs. (3) and (4) for circular radiation and is obtained for example by representing the electronic structure of the final band states as well as the initial core states in terms of the corresponding Green function [55].

The corresponding XMCD signal $\Delta \mu(\omega)=\frac{1}{2}\left(\mu_{+}(\omega)-\right.$ $\left.\mu_{-}(\omega)\right)$ is defined as the difference in absorption for left and right circularly polarized radiation. Expressing the absorption coefficient $\mu_{\lambda}(\omega)$ in terms of the absorptive part of the

TABLE I. Spin and orbital magnetic moments of $\mathrm{Mn}_{3} \mathrm{Ir}$, atomic type resolved as well as total values are given in units of $\mu_{\mathrm{B}}$. Their directions are given in the last column.

\begin{tabular}{lrrc}
\hline \hline & $\mu_{\text {spin }}\left(\mu_{\mathrm{B}}\right)$ & $\mu_{\text {orb. }}\left(\mu_{\mathrm{B}}\right)$ & $\hat{\mu}$ \\
\hline $\mathrm{Mn}$ & 2.849 & 0.066 & $\langle 112\rangle$ \\
$\mathrm{Ir}$ & -0.001 & -0.033 & {$[111]$} \\
tot. & -0.001 & -0.033 & {$[111]$} \\
\hline \hline
\end{tabular}

TABLE II. Anomalous and spin Hall conductivities, $\mathrm{AHC}=\sigma_{x y}$ and $\mathrm{SHC}=\sigma_{x y}^{z}$, respectively, for pure cubic $\mathrm{Mn}_{3} X$ compounds with $X=\mathrm{Rh}, \mathrm{Ir}$, and $\mathrm{Pt}$ in units of $(\Omega \mathrm{cm})^{-1}$. The $z$ direction corresponds to the cubic [111] direction perpendicular to the kagome planes. Results are obtained using the Kubo-Středa formula.

\begin{tabular}{lcc}
\hline \hline & $\mathrm{AHC}$ & $\mathrm{SHC}$ \\
\hline $\mathrm{Mn}_{3} \mathrm{Rh}$ & -85 & -125 \\
$\mathrm{Mn}_{3} \mathrm{Ir}$ & -280 & -230 \\
$\mathrm{Mn}_{3} \mathrm{Pt}$ & -360 & -250 \\
\hline \hline
\end{tabular}

corresponding conductivity tensor element $[12,56]$ :

$$
\mu_{\lambda}(\omega)=\frac{4 \pi}{c} \sigma_{\lambda}^{1}(\omega),
$$

with $\sigma_{\lambda}$ given by

$$
\sigma_{ \pm}(\omega)=\sqrt{1 / 2}\left(\sigma_{\mathrm{xx}}(\omega) \pm i \sigma_{\mathrm{xy}}(\omega)\right)
$$

one sees immediately that the occurrence of the off-diagonal element $\sigma_{\mathrm{xy}}(\omega)$ implies the occurrence of a XMCD signal $\Delta \mu(\omega)$. Here one should remark that Eq. (8) follows if one represents right and left circular polarization $(\lambda= \pm 1)$ as a superposition of linear polarizations according to $x \pm i y$ [56]. Alternatively, diagonalizing the conductivity tensor as given for example by Eq. (9) the resulting diagonal elements refer in the same sense to left and right circular as well as $z$ linear polarization.

\section{CUBIC $\mathrm{Mn}_{3}$ Ir}

The structure and magnetic configuration of $\mathrm{Mn}_{3} \mathrm{Ir}$ is given in Fig. 1 showing a noncollinear antiferromagnetic spin arrangement.

The Mn moments are lying in the $\{111\}$ planes of the underlying $\mathrm{Cu}_{3} \mathrm{Au}\left(\mathrm{L1}_{2}\right)$ lattice and are oriented along the $\langle 112\rangle$ directions, forming a kagome lattice of corner-sharing triangles. Calculated values for spin and orbital moments are given in Table I.

Obviously, the magnetic moment of the Mn atoms is dominated by the spin contribution. For Ir on the other hand the very small induced moment is primarily due to its orbital part.

The magnetic structure leads to the magnetic space group $R \overline{3} m^{\prime}$ that has to be considered instead of the space group $P m \overline{3} m$ (221) of the $\mathrm{Cu}_{3} \mathrm{Au}$ structure. The magnetic point group corresponding to $R \overline{3} m^{\prime}$ is $\overline{3} m^{\prime}$ and independent of the definition used for the magnetic Laue group, this implies the following shape of the conductivity tensor:

$$
\underline{\sigma}(\omega)=\left(\begin{array}{ccc}
\sigma_{x x}(\omega) & \sigma_{x y}(\omega) & 0 \\
-\sigma_{x y}(\omega) & \sigma_{x x}(\omega) & 0 \\
0 & 0 & \sigma_{z z}(\omega)
\end{array}\right) .
$$

Here the indices refer to a coordinate system that conforms with the symmetry of the system having the $z$ axis along the conventional [111] direction of the cubic $\mathrm{Cu}_{3} \mathrm{Au}\left(\mathrm{L1}_{2}\right)$ lattice, while the $x$ and $y$ axes lie in the $\{111\}$ plane. Strictly spoken, our self-consistent calculations give the Mn magnetic moment slightly tilted from the $\{111\}$ plane by $\Delta \theta=0.03^{\circ}$. This small tilting does not change the symmetry of the system 

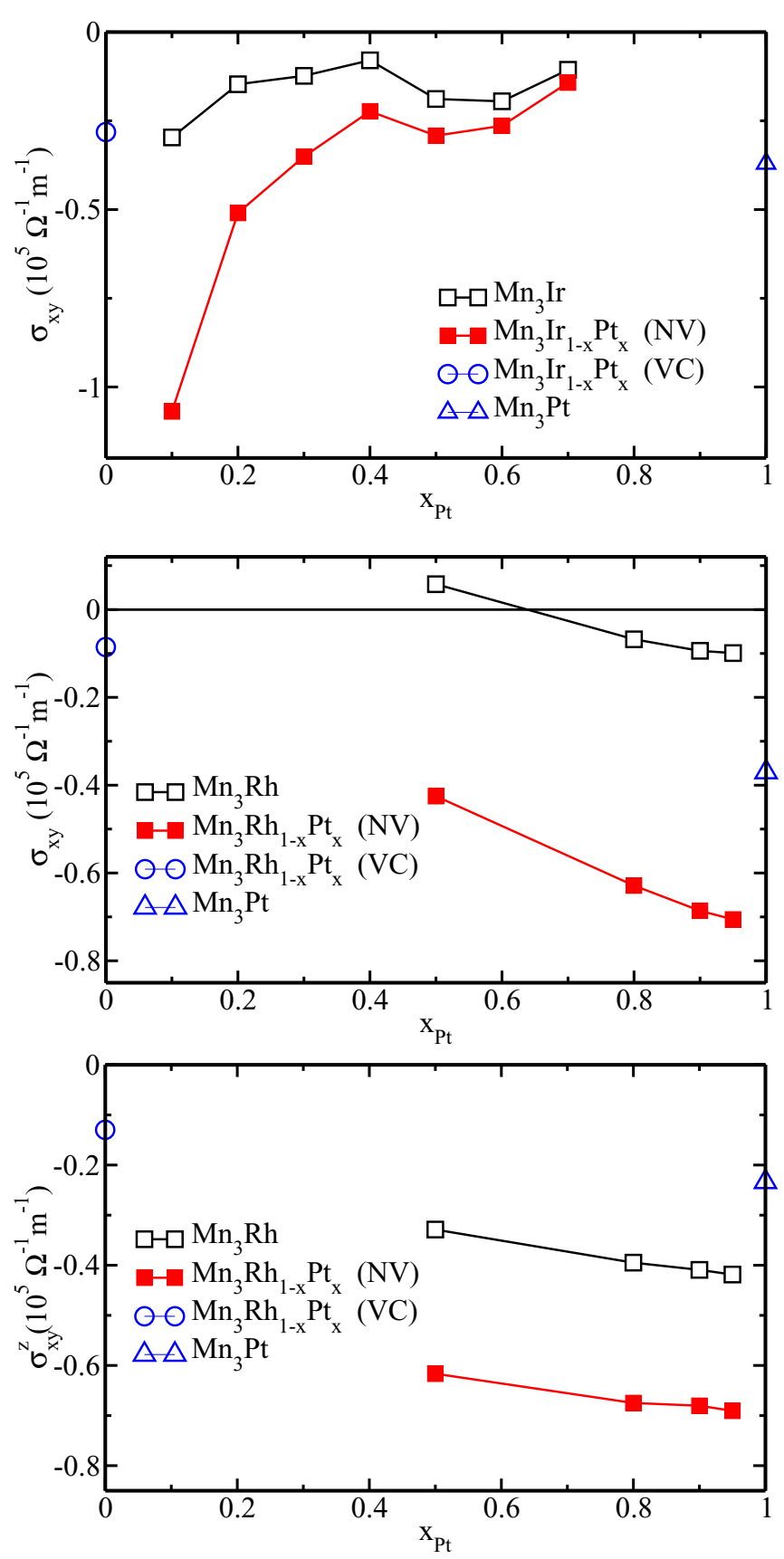

FIG. 2. Anomalous Hall conductivity of $\mathrm{Mn}_{3} \mathrm{Ir}_{1-x} \mathrm{Pt}_{x}$ (top) and $\mathrm{Mn}_{3} \mathrm{Rh}_{1-x} \mathrm{Pt}_{x}$ (middle) as well as spin Hall conductivity of $\mathrm{Mn}_{3} \mathrm{Rh}_{1-x} \mathrm{Pt}_{x}$ alloys (bottom) as a function of concentration $x$. Open symbols are results excluding vertex corrections (NV) and filled symbols such including them (VC), see text for details. The intrinsic values of the pure compounds are shown in addition. All results were obtained using the Kubo-Středa formula.

and has only little influence on the transport properties. For that reason it was not taken into account when performing the transport calculations.

Obviously, $\sigma(\omega)$ has exactly the same shape as any fcc or bcc ferromagnetic material with the magnetization along the $z$ axis that coincides with the [001] direction [12]. This implies that any gyromagnetic and magneto-optical phenomena occurring for this well-known situation will also be present for
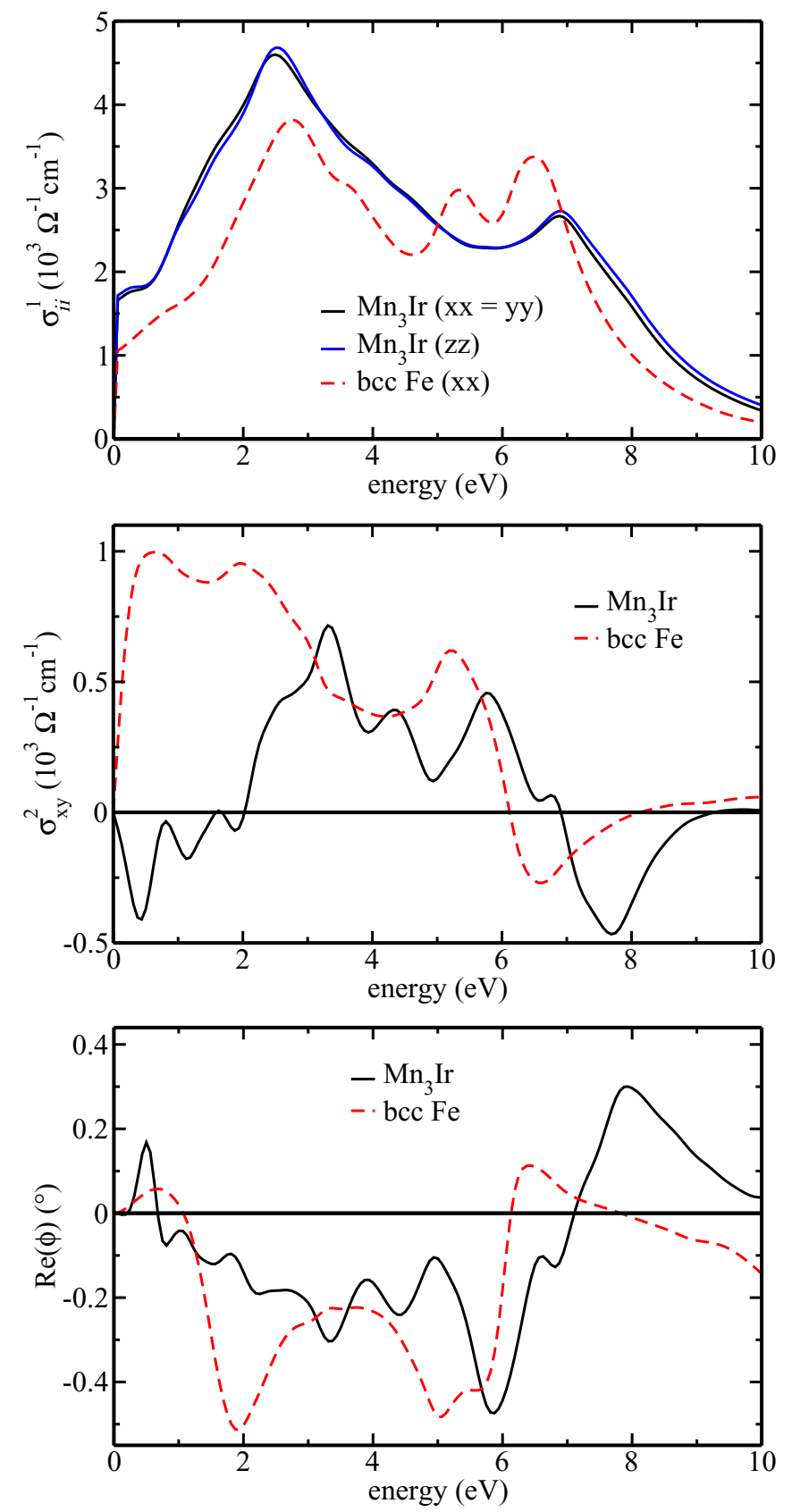

FIG. 3. Absorptive part of the optical conductivity tensor elements $\sigma_{\mathrm{xx}}^{1}, \sigma_{z z}^{1}$ (top) and $\sigma_{\mathrm{xy}}^{2}$ (middle) of $\mathrm{Mn}_{3} \mathrm{Ir}$ (full lines) together with the Kerr rotation angle $\theta_{\mathrm{K}}$ (bottom). In addition, corresponding results for ferromagnetic bcc-Fe are given (dashed lines).

the noncollinear antiferromagnet $\mathrm{Mn}_{3} \mathrm{Ir}$. For the corresponding spin conductivity tensor shapes see Ref. [26].

\section{A. Anomalous and spin Hall effect}

In the DC limit $(\omega=0)$ the conductivity tensor $\sigma(\omega)$ given by Eq. (9) becomes real. For pure systems the diagonal elements representing longitudinal conductivity diverge for $T=0 \mathrm{~K}$, while the off-diagonal anomalous Hall conductivity (AHC) $\sigma_{\mathrm{xy}}=-\sigma_{\mathrm{yx}}$ stays finite. Chen et al. [17] calculated the AHC using an expression in terms of the Berry curvature. 

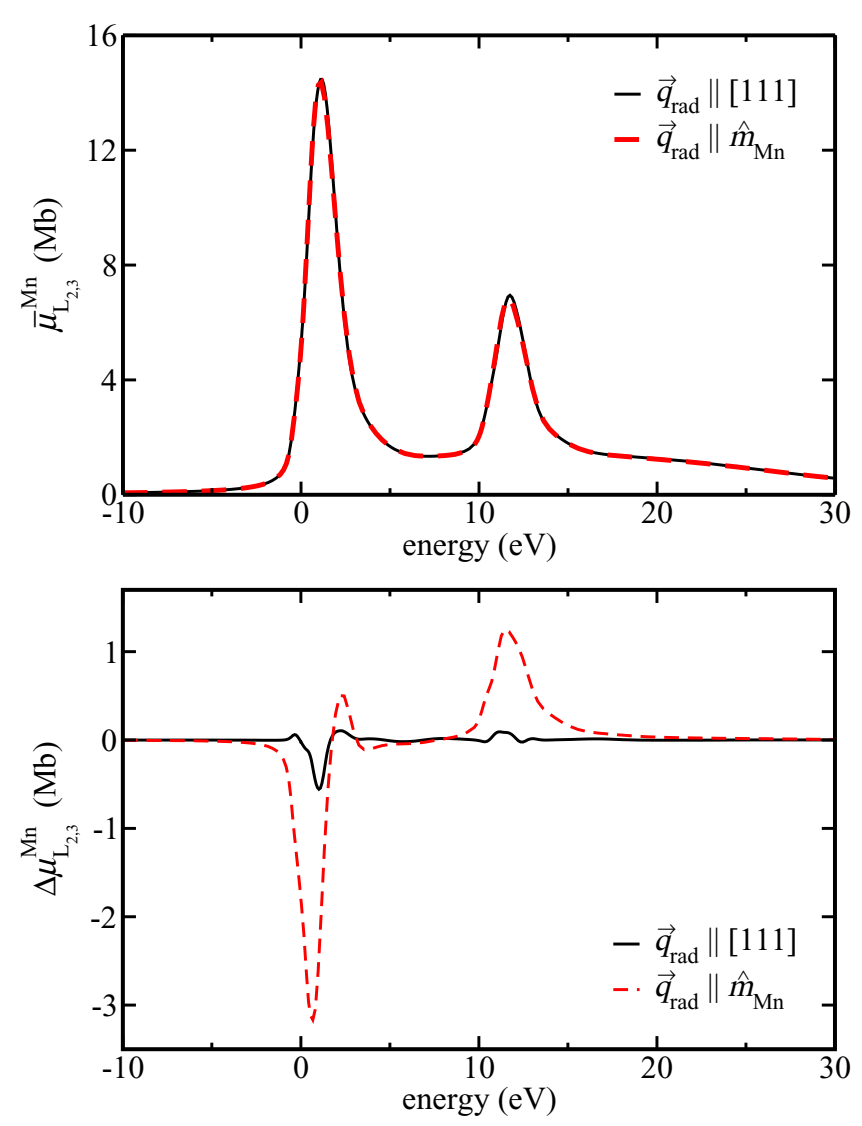

FIG. 4. Total absorption coefficient $\bar{\mu}$ at the $\mathrm{L}_{2,3}$ edge of a single $\mathrm{Mn}$ atom of noncollinear antiferromagnetic $\mathrm{Mn}_{3} \mathrm{Ir}$ (top) and corresponding circular dichroism spectra $\Delta \mu$ (bottom), both for incidence $\vec{q}_{\text {rad }}$ perpendicular (along [111]) and polar w.r.t. the magnetization direction.

Their result $\sigma_{\mathrm{AH}}=218(\Omega \mathrm{cm})^{-1}$ is comparable in size with that for the ferromagnets $\mathrm{Fe}, \mathrm{Co}$, and $\mathrm{Ni}$. In the present work the Kubo-Středa linear response formalism [48,51,58] was used, that for pure systems is completely equivalent to the Berry curvature approach.

The values obtained for the intrinsic anomalous and spin Hall conductivities at $T=0 \mathrm{~K}$ in pure $\mathrm{Mn}_{3} X$ with $X=\mathrm{Ir}$, $\mathrm{Pt}$, and $\mathrm{Rh}$ using the Kubo-Středa formula are collected in Table II.

For the $\mathrm{AHC}$ in $\mathrm{Mn}_{3} \mathrm{Ir}$ reasonable agreement with previous results by Chen et al. [17] [|218| $(\Omega \mathrm{cm})^{-1}$ ] and Zhang et al. [28] $\left[-312(\Omega \mathrm{cm})^{-1}\right]$ is achieved. The clear trend of increasing $\mathrm{AHC}$ as well as SHC with atomic number is however in disagreement with the findings of the latter authors.

Figure 2 shows results for the concentration dependence of the AHC in $\mathrm{Mn}_{3} \mathrm{Ir}_{1-x} \mathrm{Pt}_{x}$ (top) as well as for AHC and SHC in $\mathrm{Mn}_{3} \mathrm{Rh}_{1-x} \mathrm{Pt}_{x}$ (middle and bottom, respectively). While for $\sigma_{x y}$ in $\mathrm{Mn}_{3} \mathrm{Ir}_{1-x} \mathrm{Pt}_{x}$ the usual divergence of the values including vertex corrections is found, followed by a nontrivial concentration dependence, in $\mathrm{Mn}_{3} \mathrm{Rh}_{1-x} \mathrm{Pt}_{x}$ the impact of these is large for the whole investigated range. The spin Hall conductivity in this system behaves very similar to its charge counterpart. The fact that both conductivities without vertex corrections (NV) do not clearly converge towards the intrinsic values when approaching the dilute limits $x \rightarrow 0 / 1$ still has
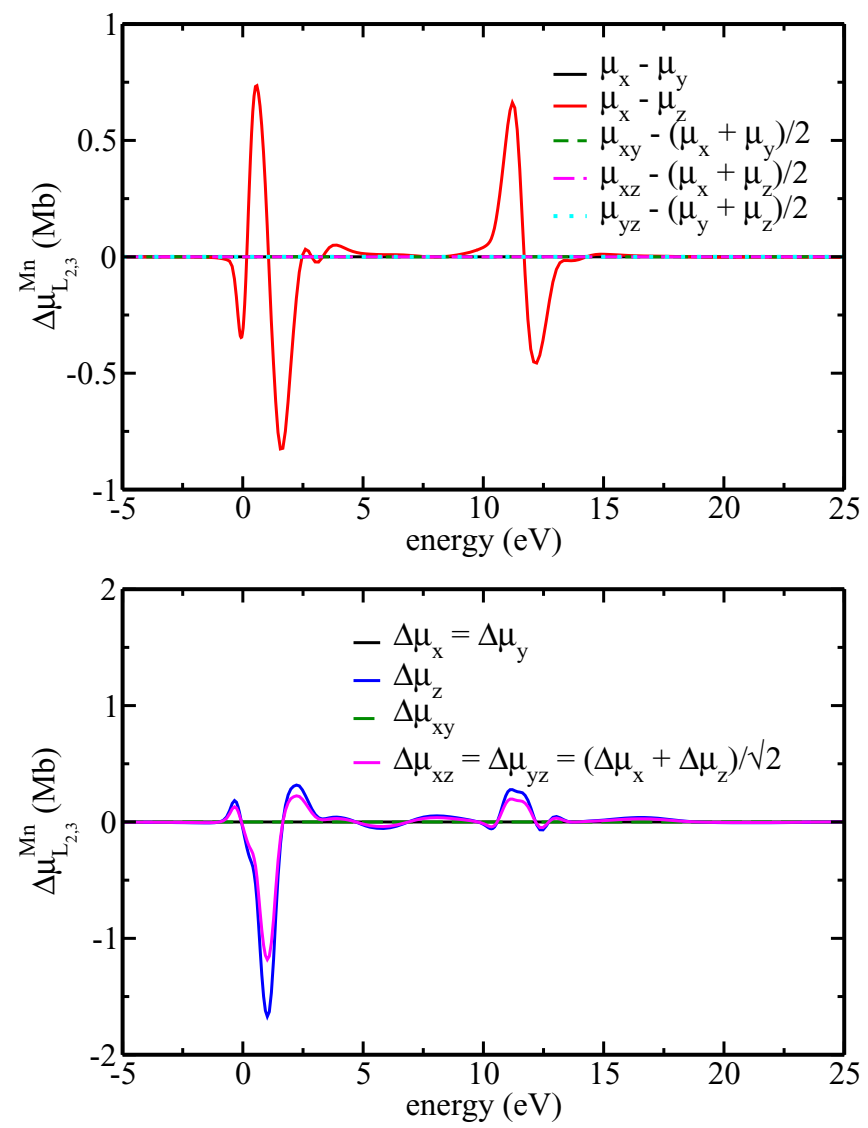

FIG. 5. Top: Differential polarization-averaged x-ray absorption spectra $\Delta \bar{\mu}$ comparing incidence along high-symmetry directions in $\mathrm{Mn}_{3}$ Ir. Bottom: X-ray magnetic circular dichroism (XMCD) spectra $\Delta \mu$ in $\mathrm{Mn}_{3} \mathrm{Ir}$ for incidence along the same directions as in the top panel.

to be clarified. For AHC and SHC in $\mathrm{Mn}_{3} \mathrm{Rh}_{1-x} \mathrm{Pt}_{x}$ an almost linear increase with increasing concentration of the heavier alloy partner Pt can be observed, the intrinsic contribution (NV) in particular for the AHC however appears to behave again nontrivially for intermediate concentrations.

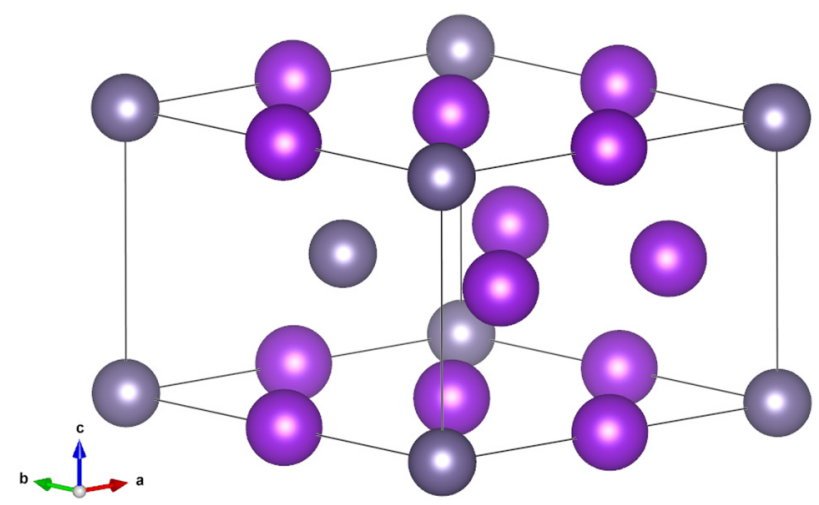

FIG. 6. Nonmagnetic unit cell of $\mathrm{Mn}_{3} \mathrm{Ge}$ in space group $P 6_{3} / m m c$ (194). Mn atoms on the Wyckoff positions $6 h$ are colored in magenta (dark gray) and Ge atoms (Wyckoff positions $2 h$ ) are colored in light gray [57]. 


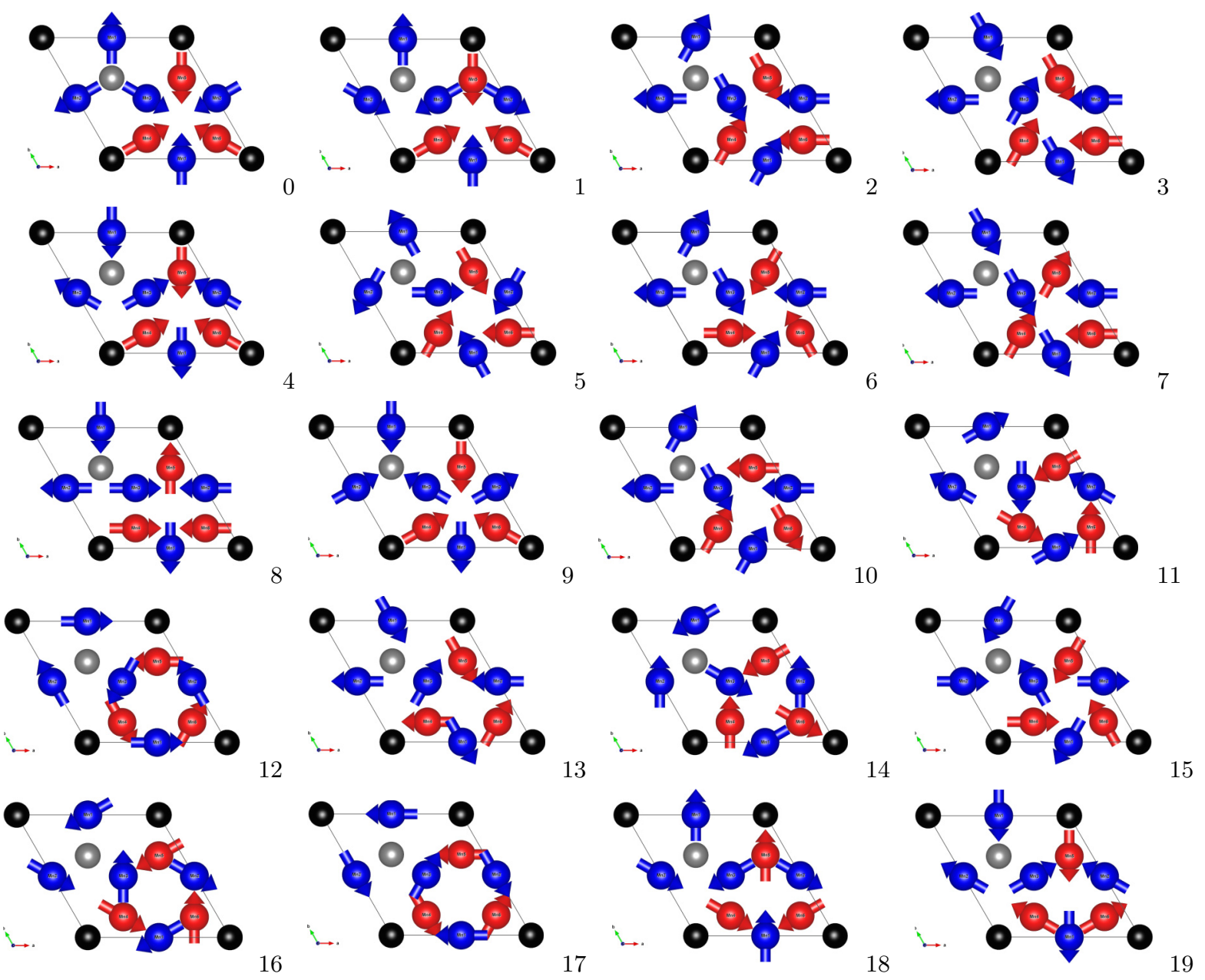

FIG. 7. Antiferromagnetic structures of $\mathrm{Mn}_{3} \mathrm{Ge}, \mathrm{AFM} i$ with $i=0 \ldots 19$, discussed in this contribution [57].

\section{B. Magneto-optical Kerr effect (MOKE)}

For finite frequencies the absorptive part of the corresponding optical conductivity tensor $\sigma(\omega)$ has been calculated in the energy regime $\hbar \omega=0-10 \mathrm{eV}$ using the fully relativistic LMTO band structure method [45-47]. Figure 3 (top) shows the corresponding real parts of the optical conductivity, $\sigma_{i i}^{1}(\omega)$. A reasonable qualitative agreement with earlier theoretical work by Feng et al. [25] is found. The small difference between $\sigma_{x x}^{1}(\omega)=\sigma_{y y}^{1}(\omega)$ and $\sigma_{z z}^{1}(\omega)$ obviously reflects the anisotropy of the system due to the underlying lattice structure as well as the magnetic ordering. Accordingly it will give rise to corresponding magneto-optical phenomena [12]. As a reference, Fig. 3 (top) gives also corresponding results for ferromagnetic bcc-Fe. In this case the anisotropy, i.e., the difference between $\sigma_{x x}^{1}(\omega)$ and $\sigma_{z z}^{1}(\omega)$, is only due to the magnetic ordering. As it is much less pronounced than for $\mathrm{Mn}_{3} \operatorname{Ir}$ only $\sigma_{x x}^{1}(\omega)$ is given.

Figure 3 (middle) shows the imaginary part of the offdiagonal transverse-optical conductivity, $\sigma_{x y}^{2}$, that is the counterpart to the $\mathrm{AHC}$ and that in particular gives rise to the polar Kerr rotation. Interestingly, $\sigma_{\mathrm{xy}}^{2}$ for $\mathrm{Mn}_{3} \mathrm{Ir}$ is in the same order of magnitude as for ferromagnetic Fe. Accordingly one finds $\theta_{\mathrm{K}}$ of $\mathrm{Mn}_{3} \mathrm{Ir}$ and $\mathrm{Fe}$ to be comparable in magnitude (see bottom panel of Fig. 3). Again reasonable qualitative agreement with Ref. [25] is found.

\section{X-ray absorption spectroscopy}

Figure 4 (upper panel) shows results of calculations for the X-ray absorption coefficient $\bar{\mu}=\frac{1}{2}\left(\mu_{+}+\mu_{-}\right)$at the $\mathrm{Mn} \mathrm{L}_{2,3}$ edges for polarization-averaged radiation. These spectra show the typical $L_{2,3}$ edges of a $3 d$ transition metal shifted against each other by the spin-orbit splitting of the $2 p$ core states. The two curves shown have been obtained for the radiation wave vector $\vec{q}_{\text {rad }}$ along the [111] direction and along the direction $\hat{m}_{\mathrm{Mn}}$ of the magnetic moment of one of the three equivalent $\mathrm{Mn}$ atoms in the unit cell. Similar to $\sigma_{\mathrm{xx}}^{1}$ and $\sigma_{z z}^{1}$ in the optical regime discussed above the difference is quite small, i.e., only a weak anisotropy occurs. The lower panel of Fig. 4 gives the corresponding XMCD curves $\Delta \mu$. For the polar geometry $\vec{q}_{\text {rad }} \| \hat{m}_{\text {Mn }}$ with the artificial restriction to one of the Mn sites the highest XMCD signal can be expected. Indeed for the $\mathrm{L}_{3}$ edge about $20 \%$ is found for the ratio $\Delta \mu / \bar{\mu}$. This is the typical order of magnitude found in ferromagnetic transition metals [12]. Considering instead the geometry $\vec{q}_{\mathrm{rad}} \|$ [111] with the X-ray beam perpendicular to the Mn magnetic moments, no XMCD signal is expected following the standard arguments [12]. In contrast to this, Fig. 4 (lower panel) clearly shows that there is indeed a finite XMCD present as one had to expect on the basis of Eqs. (7), (8), and (9). In addition one has to emphasize that the individual XMCD for the three $\mathrm{Mn}$ sites are identical, i.e., they do not compensate each other 
TABLE III. Magnetic space and Laue groups of the magnetic structures shown in Fig. 7, as well as of the nonmagnetic unit cell in Fig. $6(\mathrm{NM})$ and a ferromagnetic structure (FM) with all moments along the [0001] direction. The magnetic Laue groups are given following the definition introduced by the present authors [26] as well as the one used by Kleiner [23] (in parentheses). The conventional setting concerning the sequence of generators is used for the space groups and carried over to point and Laue groups [66].

\begin{tabular}{lcc}
\hline \hline Label & Magn. space group & Magn. Laue group \\
\hline NM & $P 6_{3} / m m c$ & $6 / m m m 1^{\prime}\left(6221^{\prime}\right)$ \\
FM & $P 6_{3} / m m^{\prime} c^{\prime}$ & $6 / m m^{\prime} m^{\prime}\left(62^{\prime} 2^{\prime}\right)$ \\
AFM0 & $P 6_{3} / m^{\prime} m^{\prime} c^{\prime}$ & $6 / m m m 1^{\prime}\left(6221^{\prime}\right)$ \\
AFM1 & $A m^{\prime} m^{\prime} 2$ & $m^{\prime} m^{\prime} m\left(2^{\prime} 2^{\prime} 2\right)$ \\
AFM2 & $P \overline{6}^{\prime} 2 c^{\prime}$ & $6^{\prime} / m^{\prime} m m^{\prime}\left(6^{\prime} 22^{\prime}\right)$ \\
AFM3 & $P m^{\prime}$ & $2^{\prime} / m^{\prime}\left(2^{\prime}\right)$ \\
AFM4 & $A m^{\prime} m^{\prime} 2$ & $m^{\prime} m^{\prime} m\left(2^{\prime} 2^{\prime} 2\right)$ \\
AFM5 & $P 6_{3} / m^{\prime}$ & $6 / m 1^{\prime}\left(61^{\prime}\right)$ \\
AFM6 & $P 6_{3} / m^{\prime}$ & $6 / m 1^{\prime}\left(61^{\prime}\right)$ \\
AFM7 & $A m^{\prime} a^{\prime} 2$ & $m^{\prime} m^{\prime} m\left(2^{\prime} 2^{\prime} 2\right)$ \\
AFM8 & $C m^{\prime} c^{\prime} m^{\prime}$ & $m m m 1^{\prime}\left(2221^{\prime}\right)$ \\
AFM9 & $P 6_{3}^{\prime} / m^{\prime} m^{\prime} c$ & $6^{\prime} / m^{\prime} m^{\prime} m\left(6^{\prime} 22^{\prime}\right)$ \\
AFM10 & $P m^{\prime}$ & $2^{\prime} / m^{\prime}\left(2^{\prime}\right)$ \\
AFM11 & $P 6_{3} / m^{\prime}$ & $6 / m 1^{\prime}\left(61^{\prime}\right)$ \\
AFM12 & $P 6_{3} / m^{\prime} m c$ & $6 / m m m 1^{\prime}\left(6221^{\prime}\right)$ \\
AFM13 & $C m^{\prime} m^{\prime}$ & $m m^{\prime} m^{\prime}\left(2^{\prime} 2^{\prime} 2\right)$ \\
AFM14 & $C m^{\prime} c m^{\prime}$ & $m^{\prime} m m^{\prime}\left(2^{\prime} 22^{\prime}\right)$ \\
AFM15 & $P 6_{3}^{\prime} / m^{\prime}$ & $6^{\prime} / m^{\prime}\left(6^{\prime}\right)$ \\
AFM16 & $P 6_{3}^{\prime} / m^{\prime}$ & $6^{\prime} / m^{\prime}\left(6^{\prime}\right)$ \\
AFM17 & $P 6_{3}^{\prime} / m^{\prime} m c^{\prime}$ & $6^{\prime} / m^{\prime} m m^{\prime}\left(6^{\prime} 22^{\prime}\right)$ \\
AFM18 & $C m^{\prime} c m^{\prime}$ & $m^{\prime} m m^{\prime}\left(2^{\prime} 22^{\prime}\right)$ \\
AFM19 & $C m^{\prime} c m^{\prime}$ & $m^{\prime} m m^{\prime}\left(2^{\prime} 22^{\prime}\right)$ \\
\hline \hline
\end{tabular}

as in the case of $\vec{q}_{\text {rad }} \| \hat{m}_{\mathrm{Mn}}$ but add up. Comparing the two XMCD spectra in Fig. 4 one notes that $\Delta \mu$ for $\vec{q}_{\text {rad }} \|$ [111] is very similar in shape to that for $\vec{q}_{\text {rad }} \| \hat{m}_{\mathrm{Mn}}$ but about one order of magnitude smaller in amplitude. Nevertheless, this implies that it should be possible to detect this XMCD signal in experiment provided one domain dominates in the regime exposed to the X-ray beam (see also comment by Chen et al. [17]).

In the top panel of Fig. 5 the difference in total absorption $\Delta \bar{\mu}=\bar{\mu}_{i}-\bar{\mu}_{j}$ with $\bar{\mu}_{i}=\frac{1}{2}\left(\mu_{i,+}+\mu_{i,-}\right)$ for incidence along pairs of high symmetry directions $(i, j=x, y, z)$ and along intermediate directions ( $i=x y, x z, y z)$ compared to the corresponding linear combinations of $x, y, z$ is shown. Note that in contrast to the conventional x-ray magnetic linear dichroism, here the polarization of the incoming light is not rotated. The Cartesian directions $x$ and $y$ were chosen to be in the (111) plane (indicated in gray in Fig. 1), i.e., corresponding to the crystallographic directions [112] $(x)$ and [110] $(y)$ of the cubic unit cell, while $z$ is parallel to the [111] direction (the space diagonal of the cubic unit cell shown in Fig. 1). As can be seen, the absorption is isotropic in the (111) plane $\left(\bar{\mu}_{\mathrm{x}}=\bar{\mu}_{\mathrm{y}}\right)$ but different for incidence perpendicular to it, i.e., along the [111] direction $\left(\bar{\mu}_{\mathrm{z}} \neq \bar{\mu}_{\mathrm{x}}\right)$. This agrees with the diagonal elements of the tensor in Eq. (9), since the first two elements are identical, $\sigma_{\mathrm{xx}}=\sigma_{\mathrm{yy}}$, and different from the third $\left(\sigma_{\mathrm{zz}}\right)$. Furthermore the absorption for intermediate directions
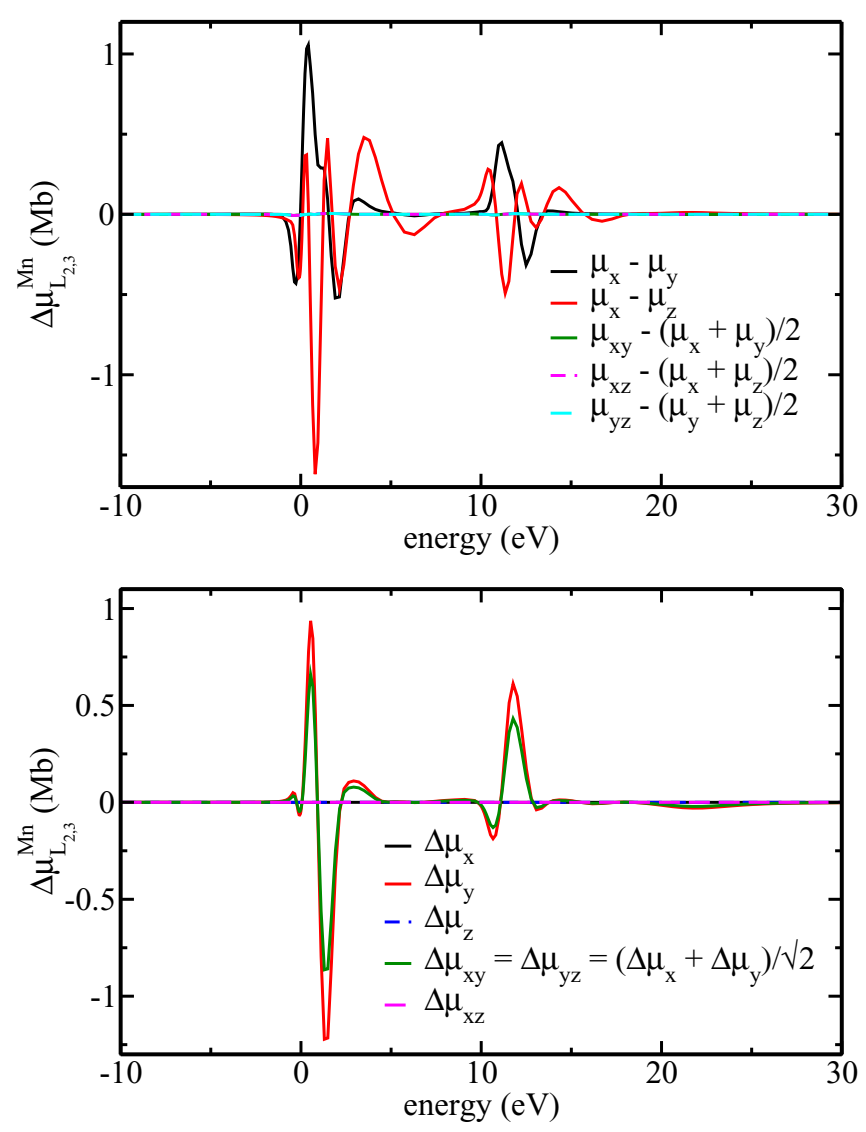

FIG. 8. Top: Differential polarization-averaged x-ray absorption spectra $\Delta \bar{\mu}$ comparing incidence along high-symmetry directions in $\mathrm{Mn}_{3} \mathrm{Ge}$ with AFM1 magnetic structure (see Fig. 7). Bottom: Corresponding $\mathrm{x}$-ray magnetic circular dichroism (XMCD) spectra $\Delta \mu$.

( $x y, x z$, and $y z$ ) can be described by a linear combination of the absorption coefficients along Cartesian axes, such as, e.g., $\bar{\mu}_{\mathrm{xz}}=\frac{1}{2}\left(\bar{\mu}_{\mathrm{x}}+\bar{\mu}_{\mathrm{z}}\right)$, i.e., no symmetric off-diagonal elements are present in the absorption tensor. In the lower panel of Fig. 5 the circular dichroism for several directions of incidence is compared. Again the results are in agreement with the tensor shape of Eq. (9), inasmuch that the only linearly independent nonzero antisymmetric tensor element is $\Delta \mu_{\mathrm{z}}$, corresponding to $\sigma_{\mathrm{xy}}=-\sigma_{\mathrm{yx}}$.

\section{HEXAGONAL $\mathrm{Mn}_{3} \mathrm{Ge}$}

\section{A. Magnetic structure and symmetry}

The hexagonal $\mathrm{Mn}_{3} \mathrm{Ge}$ compound crystallizes, as its siblings $\mathrm{Mn}_{3} \mathrm{Sn}$ and $\mathrm{Mn}_{3} \mathrm{Ga}$, in the $\mathrm{D0}_{19}$ structure with space group $P 6_{3} / m m c$ (194). The nonmagnetic unit cell is shown in Fig. 6 and will be labeled NM in the following. The Mn atoms on the Wyckoff positions $6 h$ in the $\{0001\}$ planes colored in magenta (dark gray) form triangular, so-called kagome lattices, stacked alternatingly along the [0001] $(z)$ direction. Ge atoms occupying the Wyckoff positions $2 h$ are colored in light gray.

A number of noncollinear but coplanar antiferromagnetic alignments of the moments in the two kagome sublattices have been discussed for $\mathrm{Mn}_{3} \mathrm{Ge}$ and related compounds in the 

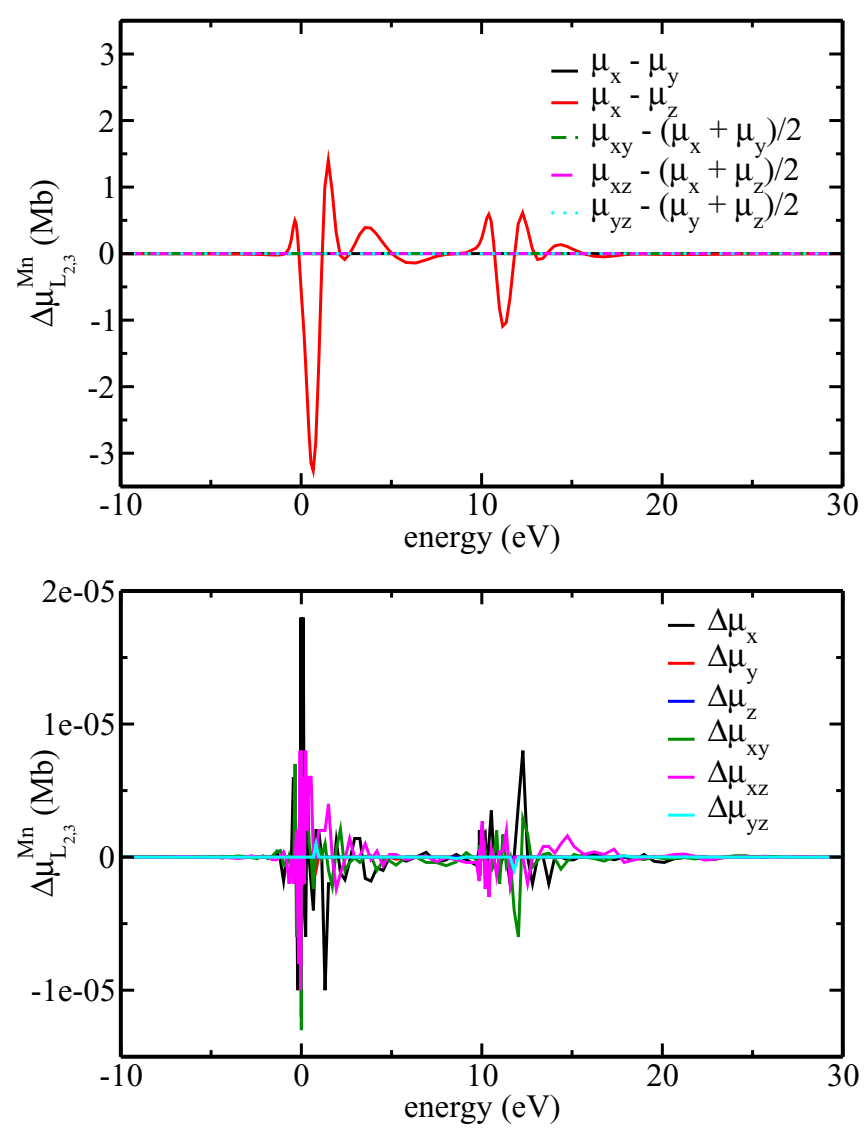

FIG. 9. Top: Differential polarization-averaged x-ray absorption spectra $\Delta \bar{\mu}$ comparing incidence along high-symmetry directions in $\mathrm{Mn}_{3} \mathrm{Ge}$ with $\mathrm{AFM} 2$ magnetic structure (see Fig. 7). Bottom: Corresponding $\mathrm{x}$-ray magnetic circular dichroism (XMCD) spectra $\Delta \mu$.

literature [18,59-63]. These are collected in Fig. 7 together with further, hypothetical spin compensated structures.

The structure AFM0 has the same spin arrangement in both kagome planes with the Mn moments pointing alternatingly towards the center of the corner-sharing triangles or away from it. The two sublattices are connected by a $\sigma_{3}$ operation, i.e., a rotation around [0001] $(z)$ by $60^{\circ}$ combined with a translation along $z$ by half of the corresponding lattice constant. This leads to a bipartite, globally chiral lattice with the nonmagnetic Laue group 6/mmm $1^{\prime}$. It corresponds to Fig. 3(a) of Ref. [63]. The structures AFM1-4 have been discussed by Kübler and Felser [18], see Figs. 2, 3(a), 3(b), and 5 therein. The structure AFM 3 is reported to be obtained by a selfconsistent calculation starting from AFM2. An anomalous Hall effect has been predicted for AFM3 and AFM4 by these authors, as well as for an additional noncoplanar structure which will not be discussed herein. The structures AFM5 and AFM6 are obtained from AFM0 by rotating all moments in the kagome planes by $30^{\circ}$, counterclockwise and clockwise, respectively. AFM7, AFM8, and AFM10 are hypothetical structures. AFM9 is obtained from AFM0 by reversing the moments in one kagome layer (here the one in blue), leading to opposite chirality in the two planes and global inversion symmetry. The sublattices are connected by $6_{3}^{\prime}$, i.e., a screw
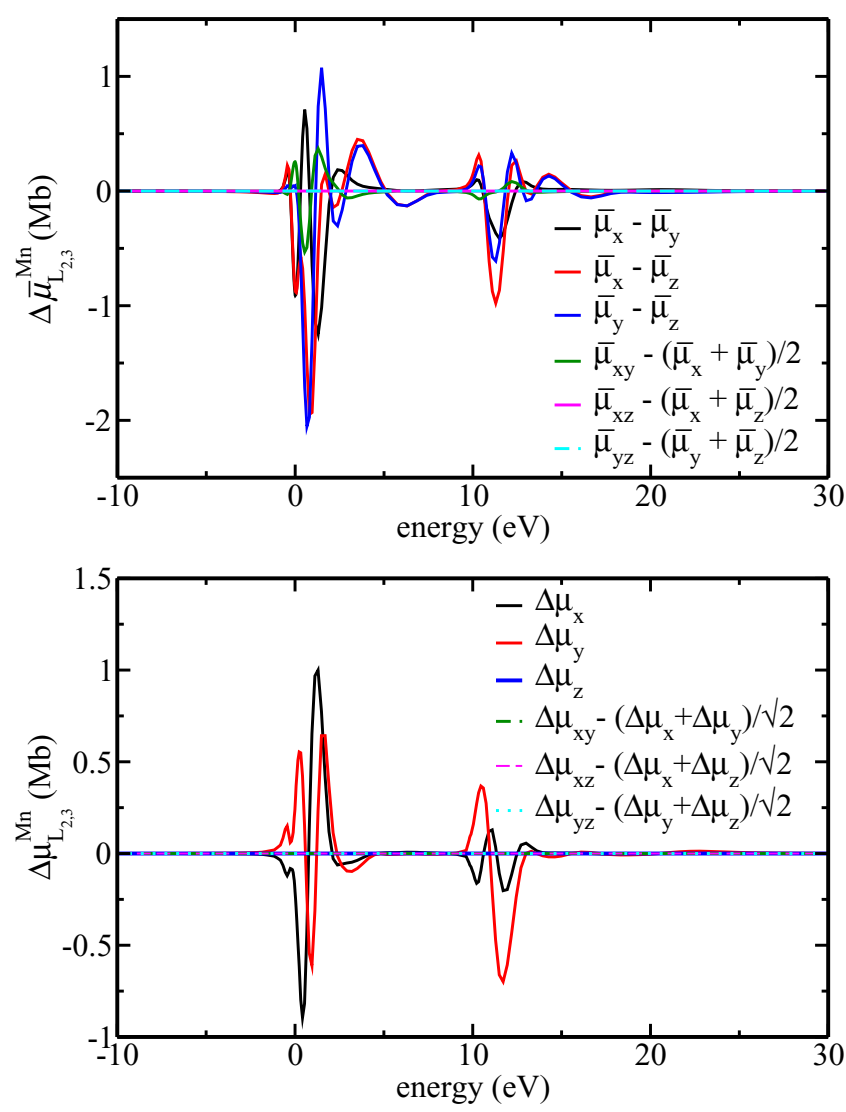

FIG. 10. Top: Differential polarization-averaged x-ray absorption spectra $\Delta \bar{\mu}$ comparing incidence along high-symmetry directions in $\mathrm{Mn}_{3} \mathrm{Ge}$ with AFM3 magnetic structure (see Fig. 7). Bottom: Corresponding x-ray magnetic circular dichroism (XMCD) spectra $\Delta \mu$.

rotation in combination with time reversal. It corresponds to Fig. 3(b) of Ref. [63]. The structures AFM11 and AFM12 are $60^{\circ}$ and $90^{\circ}$ clockwise rotations of all moments in AFM0. AFM13 and AFM14 have been proposed in Ref. [21], the first experimental verification of an anomalous Hall effect in $\mathrm{Mn}_{3} \mathrm{Ge}$. AFM13 should furthermore correspond to the magnetic structure of $\mathrm{Mn}_{3} \mathrm{Sn}$ in Ref. [20], the very first reported observation of the AHE in a noncollinear antiferromagnet of the hexagonal $\mathrm{Mn}_{3} X$ Heusler type. In AFM15, AFM16, and AFM17 the moments are $30^{\circ}, 60^{\circ}$, and $90^{\circ}$ clockwise rotated w.r.t. AFM9. Finally, AFM18 and AFM19 are proposed in Refs. [22,28-30,64]; the latter of the two has been reported by Zhang et al. [63] to be the most stable for $\mathrm{Mn}_{3} X(X=\mathrm{Ga}, \mathrm{Sn}$, $\mathrm{Ge})$ based on DFT calculations. One should stress here again that all of the above magnetic structures are coplanar and fully spin compensated, i.e., no weak ferromagnetism [65] due to an out-of-plane rotation is taken into account here.

The magnetic space and Laue groups corresponding to the structures in Figs. 6 and 7 as well as of a ferromagnetic alignment of the Mn moments along the [0001] direction are given in Table III. For convenience also the magnetic Laue group following the definition of Kleiner [23] is given in parentheses. As can be seen, a number of structures have the same magnetic space group and moreover a number of these lead to the same magnetic Laue groups. 
The corresponding symmetry-restricted tensor shapes are $[23,26]$ :

$$
\begin{gathered}
\underline{\sigma}^{\mathrm{NM}}=\left(\begin{array}{ccc}
\sigma_{x x} & 0 & 0 \\
0 & \sigma_{x x} & 0 \\
0 & 0 & \sigma_{z z}
\end{array}\right) \\
\underline{\sigma}^{\mathrm{FM}}=\left(\begin{array}{ccc}
\sigma_{x x} & \sigma_{x y} & 0 \\
-\sigma_{x y} & \sigma_{x x} & 0 \\
0 & 0 & \sigma_{z z}
\end{array}\right) \\
\underline{\sigma}^{\mathrm{AFM} 0,2,5,6,9,11,12,15,16,17}=\left(\begin{array}{ccc}
\sigma_{x x} & 0 & 0 \\
0 & \sigma_{x x} & 0 \\
0 & 0 & \sigma_{z z}
\end{array}\right) \\
\underline{\sigma}^{\mathrm{AFM} 1,4,13,18,19}=\left(\begin{array}{ccc}
\sigma_{x x} & 0 & \sigma_{x z} \\
0 & \sigma_{y y} & 0 \\
-\sigma_{x z} & 0 & \sigma_{z z}
\end{array}\right) \\
\underline{\sigma}^{\mathrm{AFM} 3,10}=\left(\begin{array}{ccc}
\sigma_{x x} & \sigma_{x y} & \sigma_{x z} \\
\sigma_{x y} & \sigma_{y y} & \sigma_{y z} \\
-\sigma_{x z} & -\sigma_{y z} & \sigma_{z z}
\end{array}\right) \\
\underline{\sigma}^{\mathrm{AFM} 7, \mathrm{AFM} 14}=\left(\begin{array}{ccc}
\sigma_{x x} & 0 & 0 \\
0 & \sigma_{y y} & \sigma_{y z} \\
0 & -\sigma_{y z} & \sigma_{z z}
\end{array}\right) \\
\underline{\sigma}^{\mathrm{AFM} 8}=\left(\begin{array}{ccc}
\sigma_{x x} & 0 & 0 \\
0 & \sigma_{y y} & 0 \\
0 & 0 & \sigma_{z z}
\end{array}\right) .
\end{gathered}
$$

In fact, for the twenty actual and hypothetical structures in Fig. 7 obviously only four different tensor shapes are found: (a) The nonmagnetic shape of Eq. (10) with the crystallographic anisotropy $\sigma_{x x}=\sigma_{y y} \neq \sigma_{z z}$ that applies for a number of antiferromagnetic structures [see Eq. (12)], (b) the ferromagnetic shape of Eq. (11) with an additional off-diagonal antisymmetric element representing the AHE. This is found again for five antiferromagnetic structures in Eq. (13) and two others in Eq. (15), only with different principal axes, (c) the full tensor with two anomalous Hall conductivities in Eq. (14), and finally (d) the diagonal fully anisotropic form of AFM8 in Eq. (16). Note that all configurations assumed in experimental (AFM13 [20,21], AFM14 [21,22], AFM18/19 [22]) as well as theoretical (AFM1-4 [18], AFM18/19 [28-30,64]) investigations on the anomalous Hall effect in hexagonal $\mathrm{Mn}_{3} X$ compounds are found to exhibit at least one off-diagonal antisymmetric element of $\underline{\sigma}$, with the exception of AFM1 for which no AHE is predicted in Ref. [18]. Corresponding tensor forms for direct and inverse spin conductivity can be found in Ref. [26], those for the spin-orbit torkance and the Edelstein polarization in chiral structures are given in Refs. [67] and [68], respectively.

\section{B. X-ray absorption spectroscopy}

The tensor shapes presented above were confirmed for selected representative cases using first-principles calculations of $\mathrm{x}$-ray absorption spectra for circularly polarized light. Figures 8-11 show for the structures AFM1-4 the difference in polarization-averaged spectra, $\Delta \bar{\mu}$, along high-symmetry
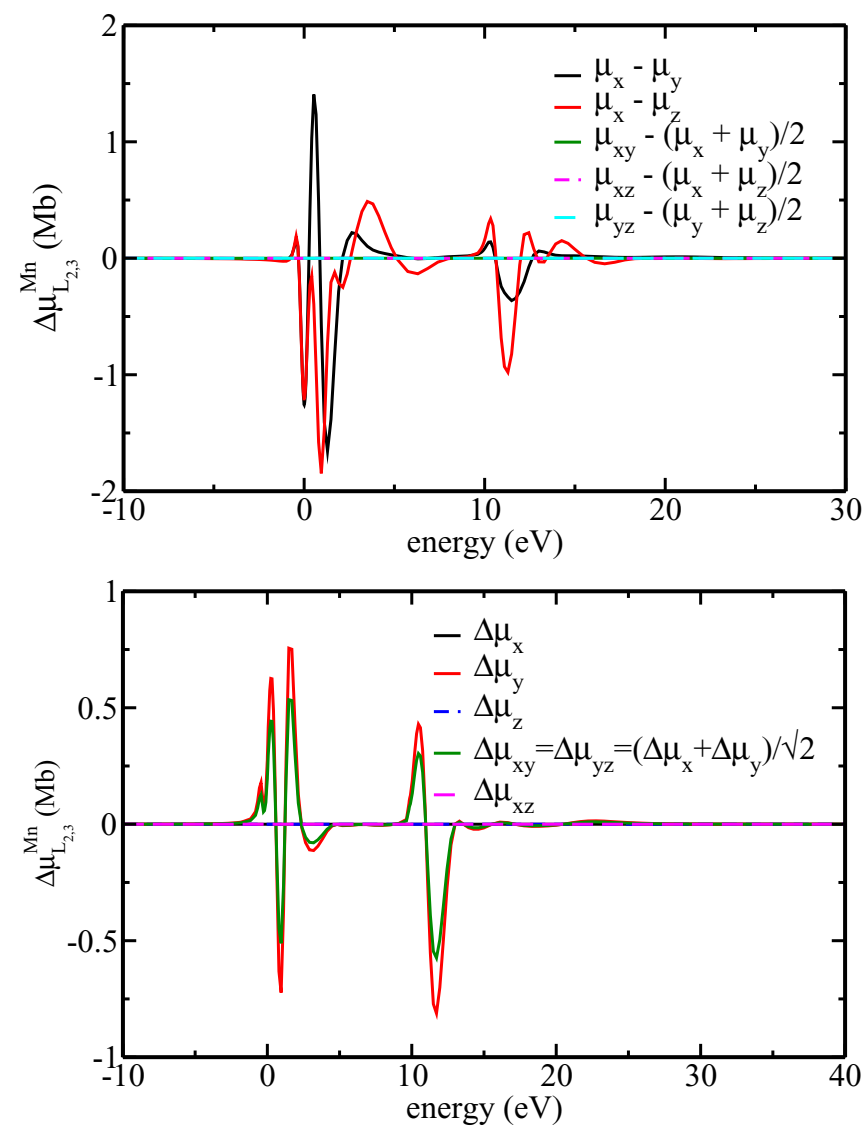

FIG. 11. Top: Differential polarization-averaged x-ray absorption spectra $\Delta \bar{\mu}$ comparing incidence along high-symmetry directions in $\mathrm{Mn}_{3} \mathrm{Ge}$ with AFM4 magnetic structure (see Fig. 7). Bottom: Corresponding $\mathrm{x}$-ray magnetic circular dichroism (XMCD) spectra $\Delta \mu$.

(Cartesian and intermediate) directions in the top panels and the corresponding spectra giving the difference in absorption for left and right circularly polarized $\mathrm{x}$ rays, the XMCD signals, in the bottom panels. As stated above (see Sec. III C), a finite $\Delta \bar{\mu}$ signal in the upper panels of Figs. 8-11 indicates an anisotropy in the symmetric part of the optical conductivity tensor, i.e., a difference on the diagonal and/or presence of symmetric contributions to off-diagonal elements. For the $\mathrm{XMCD}$ spectra in the lower panel a finite signal $\Delta \mu$ along a Cartesian direction $i$ confirms the presence of antisymmetric off-diagonal elements of $\sigma(\omega)$ with indices $j, k \neq i$. Moreover, an XMCD for incidence along intermediate directions $\langle 110\rangle$ is in all cases, if present, found to be a linear combination of the corresponding signals for incidence along the Cartesian axes. Taken together, in all four cases the predicted tensor shapes are confirmed.

\section{CONCLUSIONS}

Based on general and model-independent symmetry arguments for the occurrence of galvano-magnetic and magnetooptical phenomena in materials with arbitrary magnetic order, the implications of in particular off-diagonal antisymmetric elements of the frequency-dependent conductivity tensor in 
the coplanar noncollinear antiferromagnets $\mathrm{Mn}_{3} X$ with $X=\mathrm{Ir}$ and $\mathrm{Ge}$ have been investigated. For the cubic $\mathrm{Mn}_{3} \mathrm{Ir}$-type compound the results of first-principles Kubo linear response calculations of the anomalous and spin Hall conductivities could be shown to be in reasonable agreement with previous findings. In addition the concentration dependence of the anomalous and spin Hall effects in substitutionally disordered alloys is found to be nontrivial in particular concerning the AHE. A clear increase of the intrinsic values for both AHE and SHE, with increasing atomic number in the pure compounds $\mathrm{Mn}_{3} \mathrm{Rh}, \mathrm{Mn}_{3} \mathrm{Ir}$, and $\mathrm{Mn}_{3} \mathrm{Pt}$ is however in accordance with expectations concerning the relevance of spin-orbit coupling. The magneto-optical properties of pure $\mathrm{Mn}_{3} \mathrm{Ir}$ are again found to be in agreement with the shape of the optical conductivity tensor as well as with previous theoretical work. Comparison with corresponding results for the diagonal and off-diagonal optical conductivities in bcc Fe moreover shows that the magneto-optical Kerr effect is of comparable magnitude in both. Calculated polarization-averaged x-ray absorption and magnetic circular dichroism spectra confirm expectations concerning their symmetry and in addition the size of the XMCD signal suggests the possibility of experimental confirmation.
For the hexagonal Heusler compound $\mathrm{Mn}_{3} \mathrm{Ge}$ a number of proposed as well as additional hypothetical spin-compensated noncollinear configurations has been studied w.r.t. to magnetic symmetry and consequent electrical conductivity tensor shape. The occurrence of at least one independent anomalous Hall conductivity can be confirmed for all magnetic structures usually assumed in the experimental and theoretical literature. For selected representative cases the tensor shapes have been confirmed by supplementary x-ray absorption spectroscopy calculations. The implications of a noncoplanar spin texture in chiral and achiral magnetic structures of $\mathrm{Mn}_{3} \mathrm{Ge}$ on the linear response properties will be discussed in a separate contribution [35].

\section{ACKNOWLEDGMENTS}

Financial support by the DFG via SFB 1277 (Emergente relativistische Effekte in der Kondensierten Materie) is gratefully acknowledged. This work was further supported by the European Regional Development Fund (ERDF), project CEDAMNF, reg. no. CZ.02.1.01/0.0/0.0/15_003/0000358.
[1] E. M. Pugh and N. Rostoker, Rev. Mod. Phys. 25, 151 (1953).

[2] Y. Yao, L. Kleinman, A. H. MacDonald, J. Sinova, T. Jungwirth, D.-s. Wang, E. Wang, and Q. Niu, Phys. Rev. Lett. 92, 037204 (2004).

[3] C. Zeng, Y. Yao, Q. Niu, and H. H. Weitering, Phys. Rev. Lett. 96, 037204 (2006).

[4] N. Nagaosa, J. Sinova, S. Onoda, A. H. MacDonald, and N. P. Ong, Rev. Mod. Phys. 82, 1539 (2010).

[5] J. Kerr, Philosophical Magazine Series 5 5, 161 (1878).

[6] P. N. Argyres, Phys. Rev. 97, 334 (1955).

[7] E. Beaurepaire, J.-C. Merle, A. Daunois, and J.-Y. Bigot, Phys. Rev. Lett. 76, 4250 (1996).

[8] G. Woltersdorf, O. Mosendz, B. Heinrich, and C. H. Back, Phys. Rev. Lett. 99, 246603 (2007).

[9] H. Toyosaki, T. Fukumura, Y. Yamada, and M. Kawasaki, Appl. Phys. Lett. 86, 182503 (2005).

[10] H. Hasegawa and R. Howard, J. Phys. Chem. Sol. 21, 179 (1961).

[11] D. Y. Smith, Phys. Rev. B 13, 5303 (1976).

[12] H. Ebert, Rep. Prog. Phys. 59, 1665 (1996).

[13] J. L. Erskine and E. A. Stern, Phys. Rev. B 12, 5016 (1975).

[14] B. T. Thole, P. Carra, F. Sette, and G. van der Laan, Phys. Rev. Lett. 68, 1943 (1992).

[15] P. Carra, B. T. Thole, M. Altarelli, and X. Wang, Phys. Rev. Lett. 70, 694 (1993).

[16] P. Carra, H. König, B. Thole, and M. Altarelli, Physica B: Condensed Matter 192, 182 (1993).

[17] H. Chen, Q. Niu, and A. H. MacDonald, Phys. Rev. Lett. 112, 017205 (2014).

[18] J. Kübler and C. Felser, EPL (Europhysics Letters) 108, 67001 (2014).

[19] X. Zhou, J.-P. Hanke, W. Feng, F. Li, G.-Y. Guo, Y. Yao, S. Blügel, and Y. Mokrousov, Phys. Rev. B 99, 104428 (2019).
[20] S. Nakatsuji, N. Kiyohara, and T. Higo, Nature (London) 527, 212 (2015).

[21] N. Kiyohara, T. Tomita, and S. Nakatsuji, Phys. Rev. Applied 5, 064009 (2016).

[22] A. K. Nayak, J. E. Fischer, Y. Sun, B. Yan, J. Karel, A. C. Komarek, C. Shekhar, N. Kumar, W. Schnelle, J. Kübler, C. Felser, and S. S. P. Parkin, Sci. Adv. 2, e1501870 (2016).

[23] W. H. Kleiner, Phys. Rev. 142, 318 (1966).

[24] J. Orenstein and J. E. Moore, Phys. Rev. B 87, 165110 (2013).

[25] W. Feng, G.-Y. Guo, J. Zhou, Y. Yao, and Q. Niu, Phys. Rev. B 92, 144426 (2015).

[26] M. Seemann, D. Ködderitzsch, S. Wimmer, and H. Ebert, Phys. Rev. B 92, 155138 (2015).

[27] O. Gomonay, Phys. Rev. B 91, 144421 (2015).

[28] Y. Zhang, Y. Sun, H. Yang, J. Železný, S. P. P. Parkin, C. Felser, and B. Yan, Phys. Rev. B 95, 075128 (2017).

[29] J. Železný, Y. Zhang, C. Felser, and B. Yan, Phys. Rev. Lett. 119, 187204 (2017).

[30] Y. Zhang, J. Železný, Y. Sun, J. van den Brink, and B. Yan New J. Phys. 20, 073028 (2018).

[31] W. Zhang, W. Han, S.-H. Yang, Y. Sun, Y. Zhang, B. Yan, and S. S. P. Parkin, Sci. Adv. 2, e1600759 (2016).

[32] G.-Y. Guo and T.-C. Wang, Phys. Rev. B 96, 224415 (2017).

[33] M. Ikhlas, T. Tomita, T. Koretsune, M.-T. Suzuki, D. NishioHamane, R. Arita, Y. Otani, and S. Nakatsuji, Nat. Phys. 13, 1085 (2017).

[34] N. Nagaosa and Y. Tokura, Nat. Nanotechnol. 8, 899 (2013).

[35] S. Wimmer, S. Mankovsky, and H. Ebert, arXiv:1912.05211 (2019).

[36] W. Borchardt-Ott, Crystallography: An Introduction, 3rd ed. (Springer-Verlag, Berlin, Heidelberg, 2012), Chap. 13.4.

[37] H. T. Stokes, D. M. Hatch, and B. J. Campbell, ISOTROPY Software Suite, http://iso.byu.edu. 
[38] H. T. Stokes and D. M. Hatch, J. Appl. Crystallogr, 38, 237 (2005).

[39] A. H. MacDonald and S. H. Vosko, J. Phys. C: Solid State Phys. 12, 2977 (1979).

[40] M. E. Rose, Relativistic Electron Theory (John Wiley \& Sons, Inc., New York, London, 1961).

[41] R. Feder, F. Rosicky, and B. Ackermann, Z. Physik B 52, 31 (1983).

[42] S. H. Vosko, L. Wilk, and M. Nusair, Can. J. Phys. 58, 1200 (1980).

[43] H. Ebert, J. Braun, D. Ködderitzsch, and S. Mankovsky, Phys. Rev. B 93, 075145 (2016).

[44] H. Ebert et al., The Munich SPR-KKR package, version 7.7 (2017), http://olymp.cup.uni-muenchen.de/ak/ebert/SPRKKR.

[45] H. Ebert, Phys. Rev. B 38, 9390 (1988).

[46] A. Y. Perlov, A. N. Yaresko, and V. N. Antonov, PY-LMTO, A Spin-polarized Relativistic Linear Muffin-tin Orbitals Package for Electronic Structure Calculations (unpublished).

[47] V. Antonov, B. Harmon, and A. Yaresko, Electronic Structure and Magneto-Optical Properties of Solids (Springer, Netherlands, 2004).

[48] P. Středa, J. Phys. C: Solid State Phys. 15, L717 (1982).

[49] A. Crépieux and P. Bruno, Phys. Rev. B 64, 014416 (2001).

[50] W. H. Butler, Phys. Rev. B 31, 3260 (1985).

[51] D. Ködderitzsch, K. Chadova, and H. Ebert, Phys. Rev. B 92, 184415 (2015)

[52] C. S. Wang and J. Callaway, Phys. Rev. B 9, 4897 (1974).

[53] V. N. Antonov, A. N. Yaresko, A. Y. Perlov, and V. V. Nemoshkalenko, Low Temp. Phys. 25, 387 (1999).
[54] H. Ebert, D. Ködderitzsch, and J. Minár, Rep. Prog. Phys. 74, 096501 (2011).

[55] J. Minár and H. Ebert, Solid State Commun. 118, 383 (2001).

[56] H. S. Bennett and E. A. Stern, Phys. Rev. 137, A448 (1965).

[57] This figure has been created using the software VESTA [69].

[58] S. Lowitzer, D. Ködderitzsch, and H. Ebert, Phys. Rev. Lett. 105, 266604 (2010).

[59] E. Krén and G. Kádár, Solid State Commun. 8, 1653 (1970).

[60] T. Nagamiya, S. Tomiyoshi, and Y. Yamaguchi, Solid State Commun. 42, 385 (1982).

[61] S. Tomiyoshi and Y. Yamaguchi, J. Phys. Soc. Japan 51, 2478 (1982).

[62] S. Tomiyoshi, Y. Yamaguchi, and T. Nagamiya, J. Magn. Magn. Materials 31-34, 629 (1983).

[63] D. Zhang, B. Yan, S.-C. Wu, J. Kübler, G. Kreiner, S. S. P. Parkin, and C. Felser, J. Phys.: Cond. Mat. 25, 206006 (2013).

[64] H. Yang, Y. Sun, Y. Zhang, W.-J. Shi, S. S. P. Parkin, and B. Yan, New J. Phys. 19, 015008 (2017).

[65] L. M. Sandratskii and J. Kübler, Phys. Rev. Lett. 76, 4963 (1996).

[66] International Tables for Crystallography Volume A: Spacegroup symmetry, edited by Th. Hahn (Springer, Dordrecht, Holland, 2002).

[67] S. Wimmer, K. Chadova, M. Seemann, D. Ködderitzsch, and H. Ebert, Phys. Rev. B 94, 054415 (2016).

[68] S. Wimmer, Spin caloric transport and related phenomena from first principles, Ph.D. thesis, Munich, 2018.

[69] K. Momma and F. Izumi, J. Appl. Crystallogr. 44, 1272 (2011). 